\title{
Spontaneous coronary dissection: Optical coherence tomography insights before and after stenting
}

\section{Calin Homorodean ${ }^{1,2}$, Mihail Spinu ${ }^{1}$, Mihai Claudiu Ober ${ }^{2}$,} Maria Olinic ${ }^{1,2}$, Dan Mircea Olinic ${ }^{1,2}$

${ }^{1}$ Iuliu Hatieganu University of Medicine and Pharmacy, Cluj-Napoca, Romania

${ }^{2}$ Departament of Interventional Cardiology at Cluj County Emergency Hospital, Cluj-Napoca, Romania

A premenopausal 47-year-old female, with no significant cardiovascular risk factors, was admitted $3 \mathrm{~h}$ after the onset of an inferior ST-segment elevation myocardial infarction. Emergency coronary angiography and optical coherence tomography (OCT) imaging revealed a long, spiral, spontaneous coronary dissection (SCAD) of the right coronary artery (Fig. 1A, B and Fig. 1a-f), with a minimal

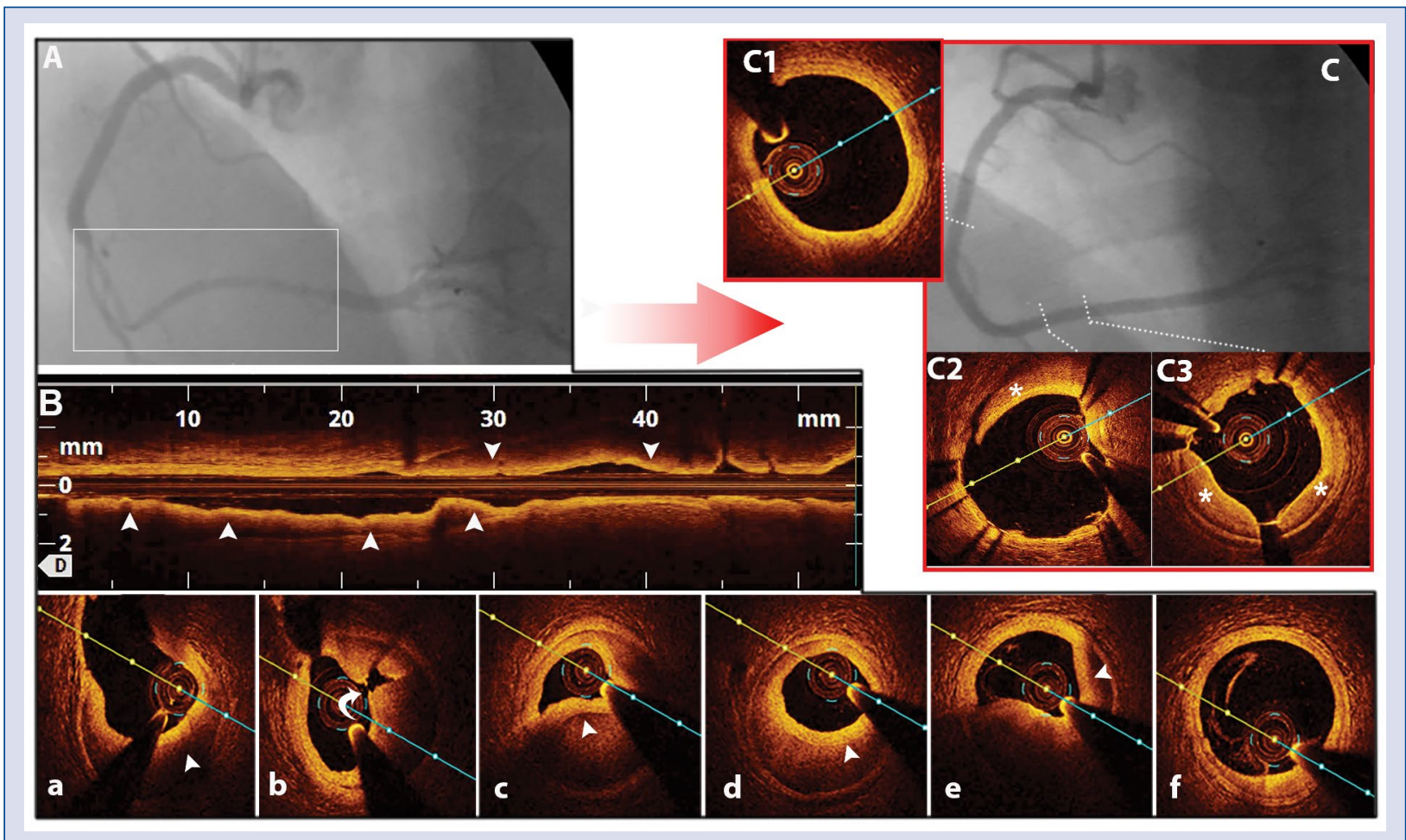

Figure 1. Right coronary artery (RCA) spontaneous dissection — diagnosis and result subsequent to stenting; $\mathbf{A}$. Coronary angiography showing severe long, tubular stenosis from mid-to-distal RCA (white box); B. Longitudinal optical coherence tomography (OCT) revealing a helicoidal intimal flap; a-f. Cross-sectional OCT showing true lumen, intimal flap and false lumen with a mixture of blood and contrast. An intimal tear is highlighted by a curved arrow in panel $b$ and intimal flap by arrowheads in panels B, a, c, d and e; C. Angiographic result after deployment of two drug-eluting stents; C1. Normal OCT aspect proximally to the stents, revealing no dissection extension; C2, C3. OCT showing the prolapse of intimal flap between stent struts (highlighted by asterisk) with a rupture depicted in C2 (asterisk).

Address for correspondence: Mihail Spinu, MD, PhD, Student; “Iuliu Hatieganu” University of Medicine and Pharmacy, Medical Clinic Number 1; 3-5, Clinicilor street, 400006, Cluj-Napoca, Romania, tel: 0040746259047 , e-mail: spinu_mihai@yahoo.com

Received: 25.10.2016 Accepted: 21.12.2016 
lumen area of $0.74 \mathrm{~mm}^{2}$. Due to ongoing angina and persistent ST-elevation in the inferior leads, a percutaneous coronary intervention (PCI) was performed, and two long everolimus-eluting stents of 3.0/38 $\mathrm{mm}$ and $3.0 / 33 \mathrm{~mm}$ were implanted with a good result (Fig. 1C). OCT control showed no proximal or distal SCAD extension (Fig. 1C1). However, tissue prolapses between the stent struts, with a base-width of $500 \mu \mathrm{m}$ and a length of $1800 \mu \mathrm{m}$ were observed (Fig. 1C2, 1C3). They could have been the result of the intimal hematoma compression during stent implantation.

In order to rule out fibromuscular dysplasia, an abdominal vascular ultrasound was performed, but no significant renal artery lesions were identified.
Patient was discharged 3 days post intervention, symptom free, on dual antiplatelet therapy.

In this case, SCAD stenting had an excellent result, although the rate of success reported in the literature is extremely variable. Intramural hematoma propagation and difficulties in advancing a coronary guidewire into the true lumen are some of the main factors usually incriminated for suboptimal PCI results. OCT guiding of the SCAD stenting procedure might improve the result by confirming guidewire position and by correctly identifying the landing zones.

Conflict of interest: None declared 